Communications in Physics, Vol. 30, No. 1 (2020), pp. 71-78

DOI:10.15625/0868-3166/30/1/14275

\title{
CHARACTERISTICS OF SIMULATED WORKPLACE NEUTRON STANDARD FIELDS
}

\section{LE NGOC THIEM $^{a, \dagger}$, NGUYEN NGOC QUYNH $^{a}$, DANG THI MY LINH ${ }^{a}$ AND PHAN THI HUONG ${ }^{b}$}

${ }^{a}$ Institute for Nuclear Science and Technology, VINATOM, 179 Hoang Quoc Viet, Hanoi, Vietnam

${ }^{b}$ Institute of Information Technology and Radiation Application, Tan Trieu, Thanh Tri, Hanoi, Vietnam

${ }^{\dagger}$ E-mail: LNThiem@vinatom.gov.vn

Received 20 August 2019

Accepted for publication 5 February 2020

Published 28 February 2020

\begin{abstract}
This paper presents the development of simulated workplace neutron standard fields at the Institute for Nuclear Science and Technology with the ${ }^{241}$ Am-Be source moderated by polyethylene spheres with diameters of $15 \mathrm{~cm}$ and $30 \mathrm{~cm}$. The characterization of the standard fields (in terms of neutron fluence rates and neutron ambient dose equivalent rates) was performed using Bonner sphere spectrometer system together with MAXED and FRUIT unfolding codes. The related quantities such as neutron dose equivalent-averaged energies and fuence-to-ambient dose equivalent conversion coefficients were also determined. The discrepancies of values are satisfied with the standard uncertainty criteria as recommended by the International Standard Organization 12789 series. It implies that the simulated workplace neutron standard fields can be applied in the practical works for calibration purposes.
\end{abstract}

Keywords: ambient dose equivalent, conversion coefficients, neutron fluence, simulated workplace neutron standard field.

Classification numbers: 06.20.fb; 87.53.Bn; 87.55.N.

\section{INTRODUCTION}

In recent years, the utilization of radiations as well as neutron sources has been rapidly increased for various applications. Therefore, accurate measurements of radiation dose equivalent rate are needed for radiation safety assessment and worker protection, which requires the establishment of neutron standard fields for calibration purposes. Recently, the Institute for Nuclear (C)2020 Vietnam Academy of Science and Technology 
Science and Technology (INST), a member in the International Atomic Energy Agency (IAEA)/ the World Health Organization (WHO) joint network of secondary standard dosimetry laboratory (SSDL) [1], possesses the unique SSDL in Vietnam for ionizing radiation dosimetry and calibration.

The International Standard Organization (ISO) has published the ISO 8529 series criteria which define the neutron standard fields with well-known neutron spectra for calibration purposes. The spectra of common neutron sources such as ${ }^{252} \mathrm{Cf}$ and ${ }^{241} \mathrm{Am}-\mathrm{Be}$ extend in the energy range from $10^{-7} \mathrm{MeV}$ to $20 \mathrm{MeV}$. However, the neutron spectra encountered at workplaces may extend in a wider energy range from $10^{-9} \mathrm{MeV}$ to $20 \mathrm{MeV}[2,3]$. Moreover, the neutron ambient dose equivalent, $H^{*}(10)$, rates measured by neutron survey meters are significantly dependent on the incident neutron spectra. Thus, if calibrations of neutron measuring devices to be used at workplaces with the ISO 8529 series standard fields become less meaningful. Therefore, the simulated workplace neutron standard fields must be created to be able to calibrate neutron measuring devices with neutron spectra similar to those at real workplaces $[3,4]$.

In this paper, polyethylene (PE) spheres with diameters of $15 \mathrm{~cm}$ and $30 \mathrm{~cm}$ were used as moderators around the ${ }^{241} \mathrm{Am}-\mathrm{Be}$ source to create the simulated workplace neutron standard fields. The characterization of these standard fields (in terms of neutron fluence rates and neutron $H^{*}(10)$ rates) has been performed using the Bonner sphere spectrometer (BSS) system combining with the MAXED and FRUIT unfolding codes [5,6]. Other quantities related to the standard fields as well as neutron dose equivalent-averaged energy and neutron fluence-to-ambient dose equivalent conversion coefficients have also been calculated.

\section{INSTRUMENT AND METHOD}

\section{II.1. Neutron calibration facility}

The neutron calibration room constructed of ordinary concrete with the $2.35 \mathrm{~g} / \mathrm{cm}^{3}$ has the inner dimensions of $700 \mathrm{~cm} \times 700 \mathrm{~cm} \times 700 \mathrm{~cm}$. The details of top view and side view are shown in a previous work [1]. The construction materials of the room were followed the data guided in the Pacific Northwest National Laboratory (i.e., PNNL-15870 Rev. 1 report) [7].

The radionuclide ${ }^{241} \mathrm{Am}-\mathrm{Be}$ neutron source of X14 type capsulation supplied by Hopewell Designs, Inc., USA was installed in a container at the base center of the calibration room. The initial source strength on January 23, 2015 is $1.299 \times 10^{7} \mathrm{~s}^{-1}$ with the expanded uncertainty of $2.9 \%(k=2)$, which is traceable to the National Institute of Standards and Technology (NIST), USA. The structure of the source is shown in the Fig. 1. When performing the measurements, the neutron source is pumped up to the center of the calibration room by a pneumatic system through a cylindrical aluminum pipe. Fig. 2 displays the neutron calibration room with the experimental arrangement.

The BSS system consists of a thermal neutron sensitive detector of ${ }^{6} \mathrm{LiI}(\mathrm{Eu})$ and a set of six polyethylene spheres with the density of $0.95 \mathrm{~g} / \mathrm{cm}^{3}$. The diameters of six polyethylene spheres are $2,3,5,8,10$, and 12 inches, respectively. The thermal neutrons are detected via a reaction

${ }^{6} \mathrm{Li}(\mathrm{n}, \alpha)^{3} \mathrm{H}(\mathrm{Q}=4.78 \mathrm{MeV})$.Then, the Ludlum 2200 scaler counts the electronic pulses from a photomultiplier. The BSS system's configuration allows detecting neutrons from thermal energy up to $20 \mathrm{MeV}$. The BSS system is basically not sensitive to photons by applying an appropriate discrimination level $[1,8,9]$. 


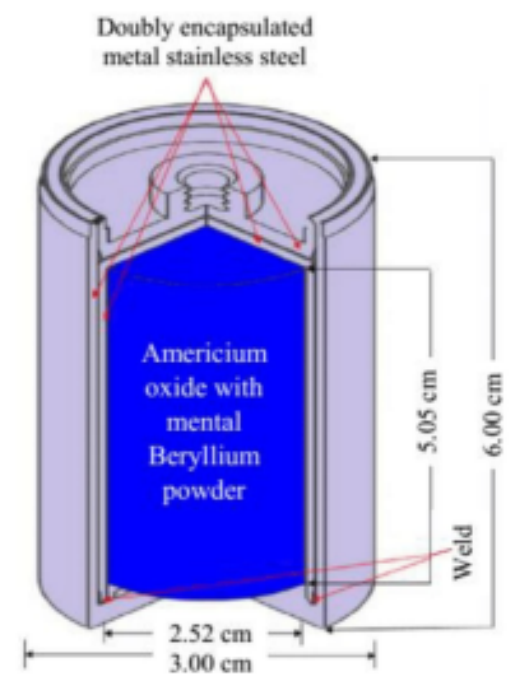

Fig. 1. ${ }^{241}$ Am-Be neutron source.

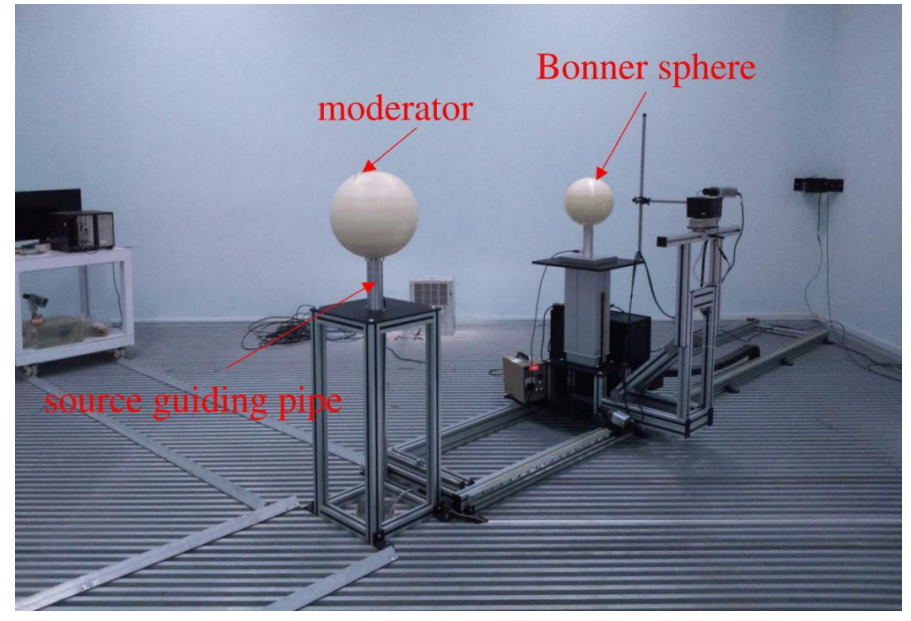

Fig. 2. Calibration room with experimental arrangement.

The radionuclide neutron sources can be moderated using a variety of moderators surrounding the sources or between the source and the detector in order to create a wider range of neutron energies and doses in the fulfillment of the practical demands on neutron measuring device calibrations. Two PE spheres (density of $0.95 \mathrm{~g} / \mathrm{cm}^{3}$ ) with diameters of $15 \mathrm{~cm}$ and $30 \mathrm{~cm}$ were used for moderating the ${ }^{241} \mathrm{Am}$-Be source for establishment of the simulated workplace neutron standard fields complying with the recommendations of ISO 12789 series [2,3]. 


\section{II.2. Experiment}

During the experiments, the BSS system is installed along a half diagonal of the room's central plane which is parallel to the base floor (see Fig. 2). The measurements of the total component of neutron fields were performed at the distances of 80,100,150,200, and $250 \mathrm{~cm}$ apart from the source center. The count rates were then used as the input data in the MAXED and FRUIT codes for unfolding the total neutron fluence rate spectra at the distances. Once, the neutron fluence rate spectrum is available, analysis is carried out to evaluate the related quantities of neutron standard fields including neutron fluence rate, neutron $H^{*}(10)$ rate, ambient dose equivalent-averaged energy, and fluence-to-ambient dose equivalent conversion coefficient $\left(h_{\phi}\right)$.

\section{II.3. Unfolding neutron spectrum}

The reading $\left(C_{i}\right)$ of the thermal detector combined with each BSS moderator sphere $i$ of a set $n$ BSS moderator spheres is the integral over a wide energy range of the product of the energy response function, $R_{i b}\left(E_{b}\right)$, of the detector $i$ and the spectral fluence, $\Psi_{b}\left(E_{b}\right)$ at energy bin $E_{b}$. The reading $C_{i}$ of the $i^{t h}$ spectrometer can be expressed as Eq. (1):

$$
C_{i}=\sum_{b=1}^{m} R_{i b}\left(E_{b}\right) \times \Psi_{b}\left(E_{b}\right) ; \quad i=1, \ldots, n ; \quad b=1, \ldots, m
$$

where $b$ is the number of energy bin; $\Psi_{b}\left(E_{b}\right)$ is the neutron fluence at the energy bin $E_{b}$. The energy response functions, $R_{i b}\left(E_{b}\right)$, were taken from the IAEA compendium [10].

The MAXED and FRUIT codes were used for unfolding the total neutron spectra from the measured data with the moderated ${ }^{241} \mathrm{Am}$-Be sources. These two codes were developed based on different unfolding algorithms. Therefore, the results obtained from two codes were compared with each other to qualify the characterization process.

The MAXED code is based on a maximum entropy principle in the inverse problem of spectrum unfolding [5]. The iterative algorithm of the MAXED code needs an initial guess spectrum which was taken from the MCNP5 simulation [11]. The FRUIT code uses the iterative algorithm of Monte Carlo method to vary the parameters and derive the final spectrum as the limit of successive spectra which fulfill the established convergence criteria [6]. No initial guess spectrum is required in the unfolding process using FRUIT. The number of energy bins used in the MAXED and FRUIT code is taken from the publication 74 of the International Commission on Radiation Protection (ICRP74) [12] for unfolding neutron spectra in the energy range from $10^{-9} \mathrm{MeV}$ to $20 \mathrm{MeV}$.

Once the neutron fluence rate spectra are determined from unfolding process, the $H^{*}(10)$ rates are calculated as Eq. (2):

$$
H^{*}(10)=\sum_{b=1}^{m} \Psi_{b}\left(E_{b}\right) \times h_{\phi, b}\left(E_{b}\right),
$$

where, $h_{\phi, b}\left(E_{b}\right)$ is the fluence-to-ambient dose equivalent conversion coefficient in the energy bin $E_{b}$ which was taken from ICRP74 [12]. 


\section{RESULT AND DISCUSSION}

\section{III.1. Neutron fluence rate spectrum}

Figure 3 depicts the neutron fluence rate spectra caused by the total components of ${ }^{241} \mathrm{Am}$ Be neutron standard fields moderated using different PE spheres at various distances from the source's center. At low energy region, the thermal neutron peaks of the spectra are appeared as the results of thermalized process. At further distances, the high neutron energy peaks decreased rapidly due to the inverse square-distance law while the thermal neutron peaks are nearly constant reflecting the constant contributions of scattered components from the room's walls and air. Comparison of the neutron fluence rate spectra obtained from the unfolding processes using MAXED and FRUIT codes shows a good agreement (see more in Fig. 3). There are some differences at the thermal energy regions. However, these differences would not contribute significantly to the integral neutron fluence rates and the $H^{*}(10)$ rates.

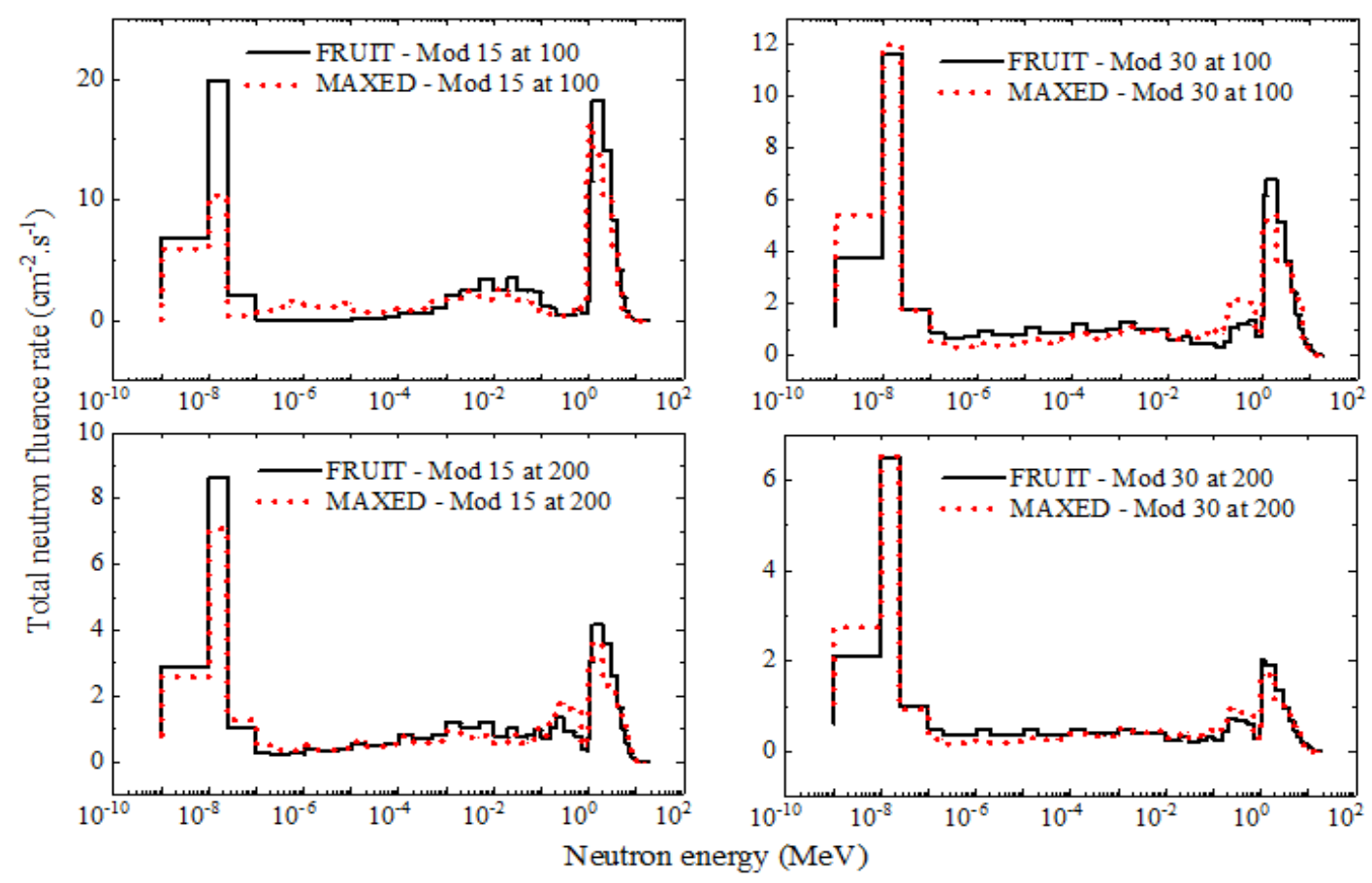

Fig. 3. Neutron fluence rate spectra (at $100 \mathrm{~cm}$ and $200 \mathrm{~cm}$ from the source's center) due to the total components of the standard fields of the ${ }^{241} \mathrm{Am}-\mathrm{Be}$ source moderated by PE spheres with diameters of $15 \mathrm{~cm}$ and $30 \mathrm{~cm}$.

\section{III.2. Neutron fluence rate and $H^{*}(10)$ rate}

Once the neutron fluence rate spectra were determined, the $H^{*}(10)$ rates can be deduced using the Eq. (2). Fig. 4 depicts the neutron fluence rate and the ambient dose equivalent rates as functions of distances from the source center. The difference in the neutron ambient dose equivalent rates and the neutron fluence rates obtained from the MAXED and FRUIT unfolding 
process are less than $8 \%$, which is satisfied the ISO 12789-1 recommendation on the standard uncertainty of the integral neutron fluence rate [2].
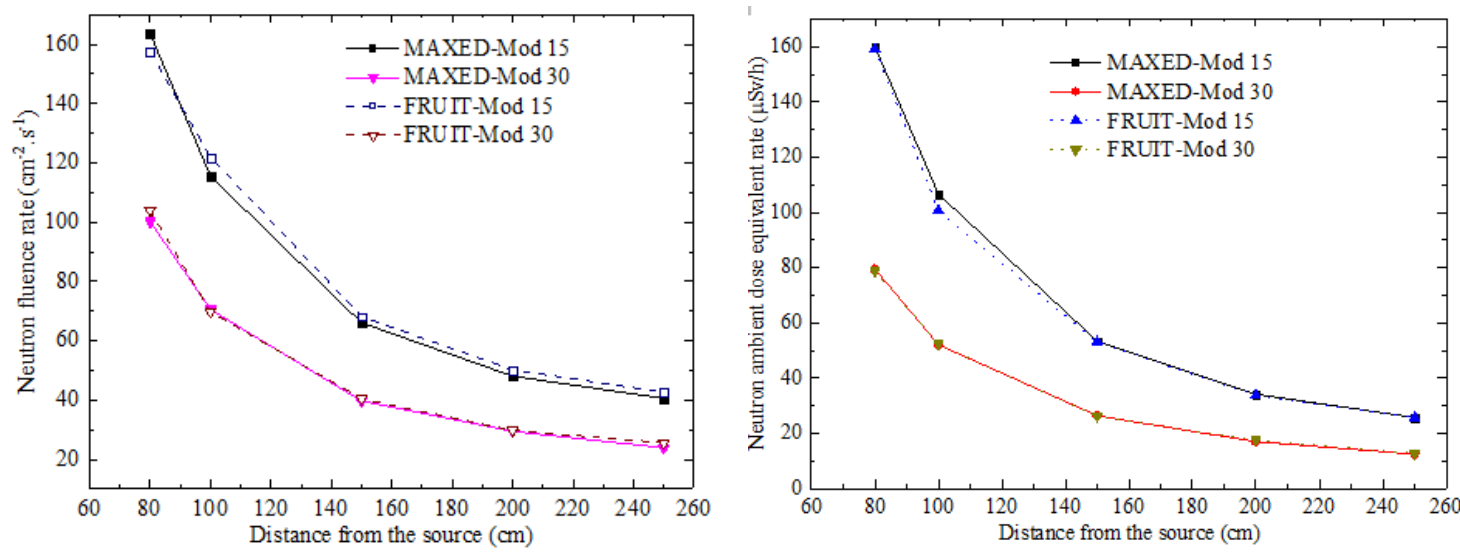

Fig. 4. Neutron fluence rate and neutron ambient dose equivalent rate obtained from different unfolding code (MAXED, FRUIT) as functions of the distances from the source moderated by PE spheres with diameters of $15 \mathrm{~cm}$ and $30 \mathrm{~cm}$.

\section{III.3. Neutron dose equivalent-averaged energy and fluence-to-ambient dose equivalent con- version coefficients}

Once the neutron fluence rates and $H^{*}(10)$ rates are available, the neutron ambient dose equivalent-averaged energy, $\widetilde{E}$, and the fluence-to-ambient dose equivalent conversion coefficients, $h_{\phi}$, can be respectively calculated as Eqs. (3)-(4):

$$
\begin{gathered}
\widetilde{E}=\frac{\sum_{b=1}^{m} H_{b}\left(E_{b}\right) \times E_{b}}{H^{*}(10)} \\
h_{\phi}=\frac{H^{*}(10)}{\sum_{b=1}^{m} \Psi_{b}\left(E_{b}\right)}
\end{gathered}
$$

Fig. 5 shows the values of $\widetilde{E}$ (left side) and $H^{*}(10)$ rates (right side) obtained from the MAXED unfolding code as functions of different neutron sources (horizontal axis). The values of $\widetilde{E}$ vary in the range of $4.4-3.1 \mathrm{MeV}$ while $H^{*}(10)$ rates reduce a factor of 3.2 from the bare source to $30 \mathrm{~cm}$ PE moderated source.

Fig. 6 depicts the values of fluence-to-ambient dose equivalent conversion coefficients, $h_{\phi}$ (left side), obtained from the MAXED and FRUIT unfolding codes. It can be seen that the values of $h_{\phi}$ vary in the respective ranges of $176-271 \mathrm{pSv} . \mathrm{cm}^{2}$ (for $15 \mathrm{~cm}$ PE moderated sphere) and 145$220 \mathrm{pSv} . \mathrm{cm}^{2}$ (for $30 \mathrm{~cm}$ PE moderated sphere). The ratio of $h_{\phi}$ between the values obtained from the MAXED code to that obtained from FRUIT code is also depicted in Fig. 3.4.The ratio varies in the range of 0.97-1.12 that means the discrepancy of $h_{\phi}$ obtained from two codes is within $12 \%$, which is satisfied with the ISO 12789-2 recommendation on the standard uncertainty of $h_{\phi}$ within $15 \%$ for the characterization process using the BSS system [3]. 


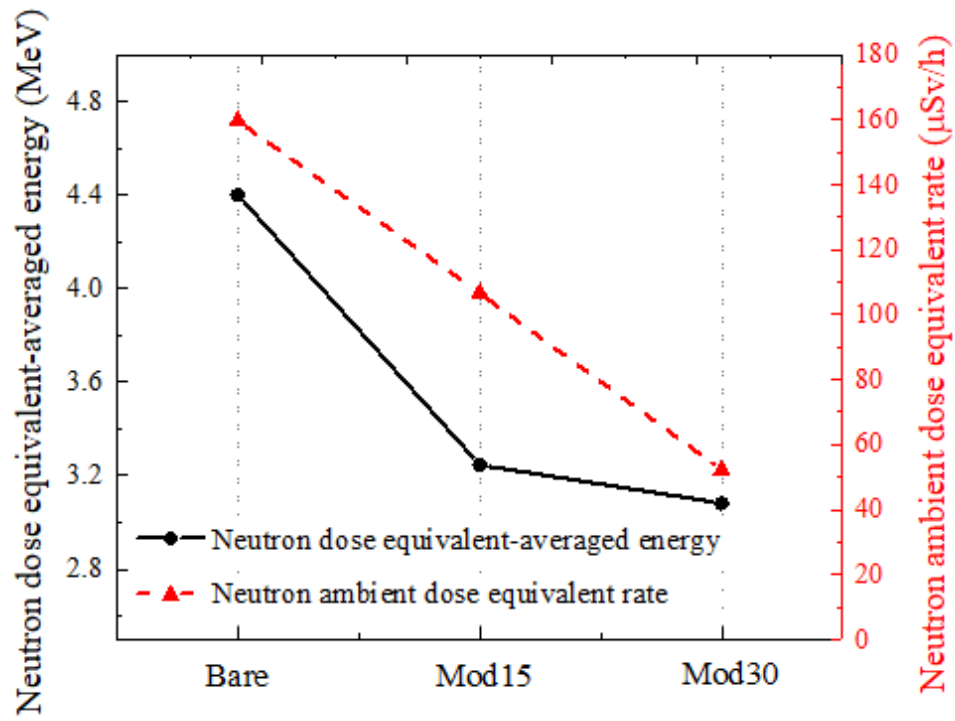

Fig. 5. Neutron ambient dose equivalent rates and dose equivalent-averaged energy at the distance of $100 \mathrm{~cm}$ for the bare ${ }^{241} \mathrm{Am}$-Be source and that moderated by two PE spheres with diameters of $15 \mathrm{~cm}$ and $30 \mathrm{~cm}$.

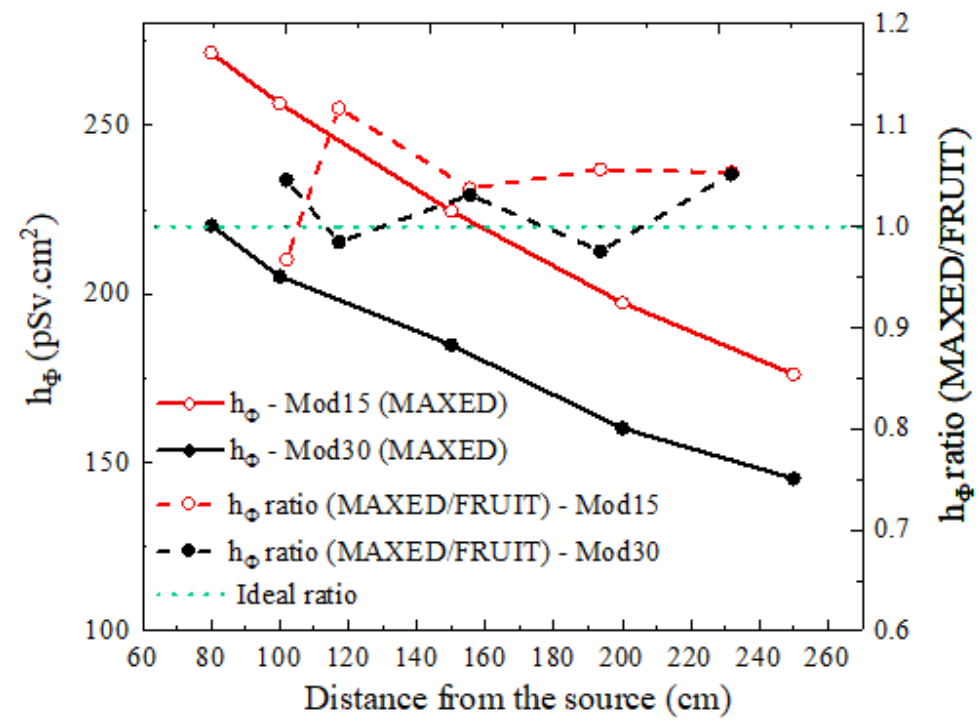

Fig. 6. Fluence-to-ambient dose equivalent conversion coefficients and their ratios between MAXED and FRUIT values as the functions of distances from the ${ }^{241} \mathrm{Am}-\mathrm{Be}$ neutron source moderated by PE spheres with diameters of $15 \mathrm{~cm}$ and $30 \mathrm{~cm}$. 


\section{CONCLUSIONS}

The simulated workplace neutron standard fields of a ${ }^{241} \mathrm{Am}$-Be source moderated by the PE spheres of $15 \mathrm{~cm}$ and $30 \mathrm{~cm}$ in diameter have been developed and characterized using the BSS system together with MAXED and FRUIT unfolding codes. The neutron fluence rates and neutron ambient dose equivalent rates were derived at five different distances from the source with the discrepancies within $8 \%$. Neutron fluence rate spectra were determined at various distances. The moderation process leads the change in neutron ambient dose equivalent-averaged energy in the range of 4.4-3.1 MeV while the neutron fluence-to-ambient dose equivalent conversion coefficients vary in the range of $145-271 \mathrm{pSv} . \mathrm{cm}^{2}$. The characterization process of neutron standard fields was verified using two unfolding codes. The results show the satisfaction with criteria recommended by ISO 12789 series. The simulated workplace neutron standard fields can be applied in the practical works for calibration purposes.

\section{ACKNOWLEDGEMENT}

The authors would like to thank National Foundation for Science and Technology Development (NAFOSTED), Viet Nam for supporting this research under the project encoded 103.042017.37. The owners of MAXED and FRUIT unfolding codes are highly appreciated for letting us using the codes.

\section{REFERENCES}

[1] L. N. Thiem, N. N. Quynh, T. V. Giap, N. T. Khai, Appl. Rad. Isot. 133 (2018) 68.

[2] ISO 12789-1:2008(E), Reference radiation fields simulated workplace neutron fields Part 1: Characteristics and methods of production, Technical Report, International Standard Organization, 2008.

[3] ISO 12789-2:2008(E), Reference radiation fields simulated workplace neutron fields Part 2: Calibration fundamentals related to the basic quantities., Technical Report, International Standard Organization, 2008.

[4] J. Chartier, B. Jansky, H. Kluge, H. Schraube and B. Wiegel, Rad. Prot. Dos. 70 (1997) 305.

[5] Environmental Measurements Laboratory, US-DOE Report EML 595 (1998), A computer code for the deconvolution of multisphere neutron spectrometer data using the maximum entropy method, US, 1998.

[6] Roberto Bedogni, Carles Domingo, Adolfo Esposito and Francisco Fernández, Nucl. Inst. Meth in Phys. Res. A 580 (2007) 1301.

[7] Pacific North West National Laboratory, NNL-15870 Rev. 1 (2011), Compendium of Material Composition Data for Radiation Transport Modeling, Washington, 2011.

[8] G. F. Knoll, Radiation detection and measurement, 4th Edition ed., Wiley, 2010.

[9] J. A. Carelli, J. L. Cruzate and B. N. Gregori, Bonner Sphere Spectrometer, https://pdfs.semanticscholar.org/4846/d4790c7fce41b4d1ccd2a0e00b7718030d0e.pdf

[10] Technical Reports Series No. 318, (2001), Compendium of Neutron Spectra and Detector Responses for Radiation Protection Purposes, International Atomic Energy Agency, Vienna, 2001.

[11] X-5 Monte Carlo Team, (2005), MCNP-A general Monte Carlo N-particle transport code, Technical Report, Version 5-Vol.II: Users Guide, Los Alamos National Laboratory, California, USA, 2005.

[12] ICRP Publication 74, (1996), Conversion coefficients for use in radiological protection against external radiation, Technical Report, Annals of the ICRP 26, 1996. 